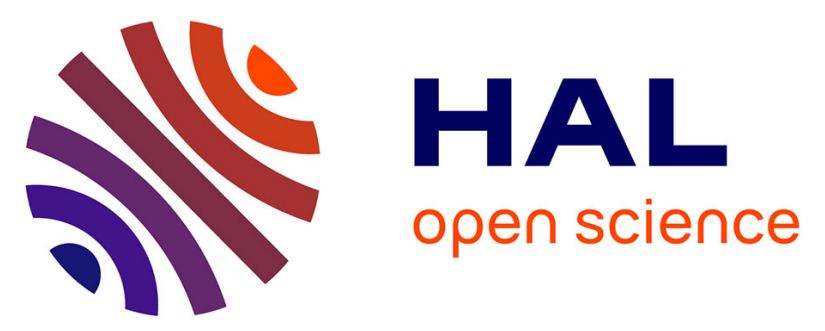

\title{
Stability of DC/DC three terminals converter using Modular Multilevel Converters for HVDC systems
}

Miguel Jiménez Jiménez Carrizosa, Gilbert Bergna, Amir Arzande, Gilney

Damm, Pedro Alou, Abdelkrim Benchaib, Françoise Lamnabhi-Lagarrigue

\section{- To cite this version:}

Miguel Jiménez Jiménez Carrizosa, Gilbert Bergna, Amir Arzande, Gilney Damm, Pedro Alou, et al.. Stability of DC/DC three terminals converter using Modular Multilevel Converters for HVDC systems. 17th European Conference on Power Electronics and Applications (EPE-ECCE Europe 2015), Sep 2015, Genève, Switzerland. 10.1109/EPE.2015.7309421 . hal-01316357

\section{HAL Id: hal-01316357 \\ https://hal.science/hal-01316357}

Submitted on 15 Sep 2020

HAL is a multi-disciplinary open access archive for the deposit and dissemination of scientific research documents, whether they are published or not. The documents may come from teaching and research institutions in France or abroad, or from public or private research centers.
L'archive ouverte pluridisciplinaire $\mathbf{H A L}$, est destinée au dépôt et à la diffusion de documents scientifiques de niveau recherche, publiés ou non, émanant des établissements d'enseignement et de recherche français ou étrangers, des laboratoires publics ou privés. 


\title{
Stability of DC/DC three terminals converter using Modular Multilevel Converters for HVDC systems.
}

\author{
M. Jiménez Carrizosa ${ }^{1}$, G. Bergna ${ }^{2}$, A. Arzandé ${ }^{3}$, G. Damm $^{4}$, P. Alou ${ }^{5}$, \\ A. Benchaib ${ }^{6}$ and F. Lamnabhi-Lagarrigue ${ }^{1}$ \\ ${ }^{1}$ LSS CentraleSupélec, France, email: Miguel.Jimenez@1ss.supelec.fr, lamnabhi@1ss.supelec.fr \\ ${ }^{2}$ SINTEF Energy Research, Norway, email: gilbert.bergna-diaz@ sintef.no \\ ${ }^{3}$ CentraleSupélec, France, email: Amir.Arzande@centralesupelec.fr \\ ${ }^{4}$ IBISC, France, email: gilney.damm@ibisc.fr \\ ${ }^{5}$ Centro de Electrónica Industrial, UPM, Spain, email: pedro.alou@upm.es \\ ${ }^{6}$ ALSTOM GRID / Cnam, France, email: abdelkrim.benchaib@alstom.com
}

\section{Keywords}

$<<$ MMC $>>,<<$ MT-HVDC $>>,<<$ DC/DC converters $>>,<<$ hub $>>,<<$ Lyapunov theory $>>$.

\begin{abstract}
This paper presents the modelling and control of a DC/DC bidirectional converter with 3 terminals based on Modular Multilevel Converter (MMC) suitable for high voltage and power applications in multi-terminals high voltage direct current (MT-HVDC) grids. A proof is given in order to guarantee the global stability of the system by means of Lyapunov theory and switching control theory. In addition, a study on how to improve the generated harmonics is shown.
\end{abstract}

\section{Introduction.}

In recent years the use of DC grids has grown significantly mainly due to several factors: current $\mathrm{AC}$ networks are reaching their limits and they are becoming overloaded, besides DC grids favour the integration of renewable energy sources, especially wind offshore. These facts together with the recent development of power electronics, and more specifically the development of converters for high voltage and high power applications, have generated a suitable framework for the development and study of MT-HVDC grids [1]. In this context it should be highlighted voltage source converters (VSC) $[1,2]$ and more recently the MMC [3](both for AC/DC conversion).

Nowadays the option of using MMC over VSC is prevailing. The advantages of using MMC technology with respect to VSC technology are several: the resulting waveform has a very small harmonic content and it has reduced transient voltage stresses and hence lower high frequency noise appears [4]. Also it has the ability to continue its operation in unbalance conditions [5].

However one of the more vital elements for the development of MT-HVDC grids is the bidirectional $\mathrm{DC} / \mathrm{DC}$ converter, the equivalent to the transformer for AC grids, which principal mission is to link networks with different DC voltages. Besides the DC/DC converter can provide other benefits, such as the use of itself as DC circuit-breaker and also to regulate the power flow in the grid [6].

One line of research for the DC / DC bidirectional converters for MT-HVDC is based on the wellknown topology Dual Active Bridge (DAB) [7, 8]. Nevertheless, and although it has optimal performances and advantages over other topologies,it uses an internal transformer at high frequency [9], which increases significantly the cost of this device. Other authors suggest to link two MMC by the alternative side in order to connect two networks with different DC voltages, as proposed [10]. This structure provides the advantage that the internal transformer is avoided, and also it offers all the advantages given by the use of MMC technology explained above. 
The main idea proposed in this paper is to implement a DC/DC bidirectional converter which operates with three terminals using MMC converters (2 phases) as shows figure 1. Consequently the DC/DC converter explain here could address the function of the $h u b$ (or common connection point) which carries out the union between several grids with different voltages[11].

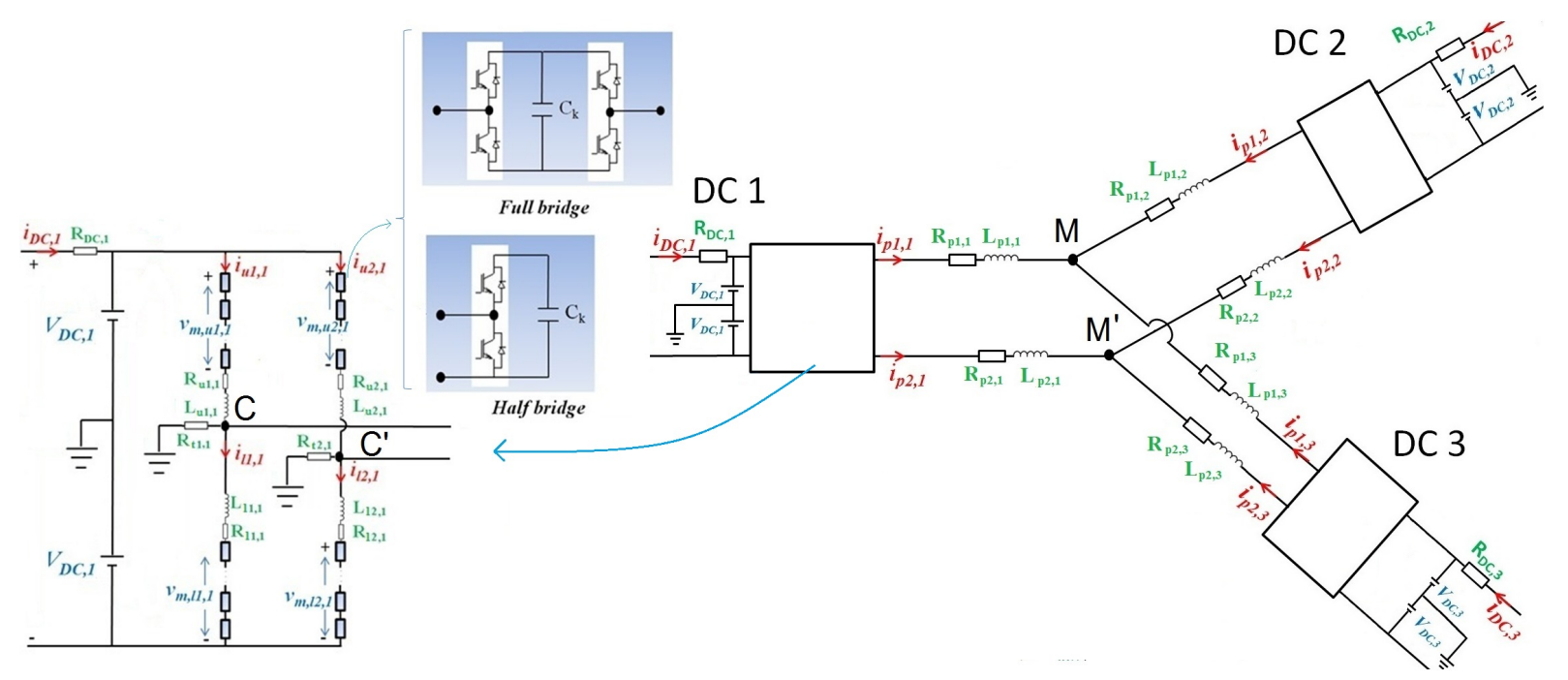

Figure 1: Three terminals DC/DC converter with 2 phases based on MMC.

This work is outlined as follows: Section II presents the modelling of a DC/DC multi-terminal converter based on MMC. In Section III the system stability is studied and the control philosophy is shown. In Section IV the simulations are carried out. Finally, in Section $\mathbf{V}$ the conclusions are explained.

\section{DC/DC multi-terminal converter based on MMC}

\subsection{MMC model.}

Each MMC phase is divided in two arms, the upper and the lower. Each arm is composed by $N$ submodules (hundreds in HVDC applications) which usually could have two different forms (see [10]): the half bridge, constituted by two switches (IGBT and diode), or the full bridge, formed by four switches as shows the figure 1 . The use of full bridges is specially interesting when a fault appears, because controlling them, we can impose the appropriate polarity of voltage during fault events in order to block the fault current [12]. However, for the purpose of this paper is sufficient with consider half bridges. On the other hand, it is important to point out that it is necessary an inductance in the arm in order to compensate the voltages imbalances between the upper and lower arms and the DC voltage. Finally, resistances $R_{t, j}$ shown in figure 1 links the arms with the earth in order to guarantee that each set of submodules achieve the voltage of the corresponding DC grid, and also they establish the required currents for the DC/DC conversion process [12].

\subsection{DC/DC multi-terminal converter model based on MMC.}

In figure 1 a DC/DC converter with 3 terminals and 2 phases based on MMC is shown. With the aim of build the model, we write the system equations. Regarding figure 1, it is true that:

$$
\begin{gathered}
\left\{\begin{array}{c}
V_{D C 1}=R_{u 1,1} \cdot i_{u 1,1}+L_{u 1,1} \cdot i_{u 1,1}+v_{u 1,1}+R_{t 1,1} \cdot i_{t 1,1}+L_{t 1,1} \cdot i_{t 1,1} \\
V_{D C 1}=R_{l 1,1} \cdot i_{l 1,1}+L_{l 1,1} \cdot i_{l 1,1}+v_{l 1,1}-R_{t 1,1} \cdot i_{t 1,1}-L_{t 1,1} \cdot i_{t 1,1} \\
\vdots \\
V_{D C 3}=R_{l k, 3} \cdot i_{l k, 3}+L_{l k, 3} \cdot i_{l k, 3}+v_{l k, 3}-R_{t k, 3} \cdot i_{t k, 3}-L_{t k, 3} \cdot i_{t k, 3}
\end{array}\right. \\
\left\{\begin{array}{c}
R_{t 1,1} \cdot i_{t 1,1}+L_{t 1,1} \cdot i_{t 1,1}=R_{p 1,1} \cdot i_{p 1,1}+L_{p 1,1} \cdot i_{p 1,1}-R_{p 1,2} \cdot i_{p 1,2}+L_{p 1,2} \cdot i_{p 1,2}+R_{t 1,2} \cdot i_{t 1,2}+L_{t 1,2} \cdot i_{t 1,2} \\
\vdots \\
R_{t 2,2} \cdot i_{t 2,2}+L_{t 2,2} \cdot i_{t 2,2}=R_{p 2,2} \cdot i_{p 2,2}+L_{p 2,2} \cdot i_{p 2,2}-R_{p 2,3} \cdot i_{p 2,3}+L_{p 2,3} \cdot i_{p 2,3}+R_{t 2,3} \cdot i_{t 2,3}+L_{t 2,3} \cdot i_{t 2,3}
\end{array}\right.
\end{gathered}
$$


In each middle point of the arms (points $C_{\text {and }} \mathrm{C}^{\prime}$ ) and in points $\mathrm{M}$ and $\mathrm{M}^{\prime}$, in the figure 1 , it is also true by means of Kirchhoff's law, prespectively that:

$$
\left\{\begin{array} { c } 
{ i _ { u 1 , 1 } = i _ { l 1 , 1 } + i _ { p 1 , 1 } + i _ { t 1 , 1 } } \\
{ i _ { u 2 , 1 } = i _ { l 2 , 1 } + i _ { p 2 , 1 } + i _ { t 2 , 1 } } \\
{ \vdots } \\
{ i _ { u 2 , 3 } = i _ { l 2 , 3 } + i _ { p 2 , 3 } + i _ { t 2 , 3 } }
\end{array} \quad \left\{\begin{array}{l}
i_{p 1,1}+i_{p 1,2}+i_{p 1,3}=0 \\
i_{p 2,1}+i_{p 2,2}+i_{p 2,3}=0
\end{array}\right.\right.
$$

From equations (3), it is clear that the order of state variable will be reduced. At this point, we will use as state variables all the currents in the upper and lower arms, in total 12 variables, and 4 state variables for the currents in each $\mathrm{AC}$ phase.

In concordance with [13], and considering the total energy stored in the arms, the dynamics of $v_{m, u i, j}$ could be expressed as $\dot{v}_{m u i, j}=\frac{N_{u i, j} \cdot n_{u i, j}}{\mathcal{C}_{u i, j}} \cdot i_{u i, j}$, where $n_{u j, s}$ and $n_{l j, s}$ are the index number for the upper and lower arm respectively, $\mathcal{C}_{u j, s}$ and $\mathcal{C}_{l j, s, s}$ are the total module capacitance for the upper and lower arms, and $N_{u j, s}$ and $N_{l j, s}$ are the number of sub-modules for the upper and lower in the $j^{t h}$ leg of the $s^{\text {th }}$ terminal. In order to achieve an optimal performance all modules must have the same number of sub-modules. In this conditions, and for the proper operation of the converter, the sum of the sum-modules in state on between the upper and lower arms in each leg must be always constant. Consequently, the sum of the upper ans lower index number must be always equals to one for all legs.

Therefore the multi-terminal converter dynamics with 2 phases and 3 terminals could be expressed, in the first instance, by the following non linear system.

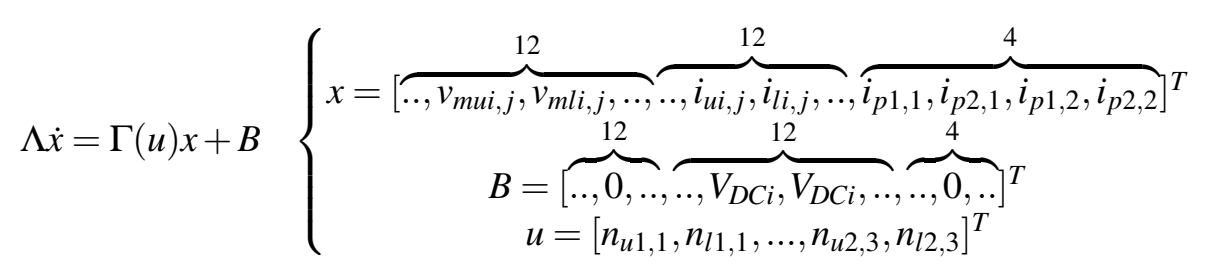

where $x, B \in \mathbb{R}^{28}$, and in $u \in \mathbb{R}^{12}$ are the control variables (which are always positive and bounded).

The matrix $\Lambda \in \mathbb{R}^{28 \times 28}$ has the following form:

$$
\Lambda=\left[\begin{array}{ll}
\Lambda_{11} & \Lambda_{12} \\
\Lambda_{21} & \Lambda_{22}
\end{array}\right]=\left[\begin{array}{cc}
\mathbf{I}_{12} & \mathbf{0} \\
\mathbf{0} & \Lambda_{22}
\end{array}\right] ; \Lambda_{22}=\left[\begin{array}{cc}
\Lambda_{22}^{a} & \Lambda_{22}^{b} \\
\left(\Lambda_{22}^{b}\right)^{T} & \Lambda_{22}^{c}
\end{array}\right] \in \mathbb{R}^{16 \times 16} ; \Lambda_{22}^{c}=\left[\begin{array}{cc}
\Sigma_{1} & S \\
S & \Sigma_{2}
\end{array}\right] \in \mathbb{R}^{4 \times 4}
$$

where $\Sigma_{s}=\operatorname{diag}\left(\sigma_{1}^{s}, . ., \sigma_{k}^{s}\right) \in \mathbb{R}^{2 \times 2}, \forall s \in\{1,2\}$, with $\sigma_{i}^{s}=L_{t i, s}+L_{p i, s}+L_{t i, n}+L_{p i, n} \forall i \in\{1,2,3\}$, and the index $n$ refers to the terminal $n$. The terms $S$ are defined as $S=\operatorname{diag}\left(. ., L_{t i, n}+L_{p i, n}, ..\right) \in \mathbb{R}^{2 \times 2}$ Matrix $\Lambda_{22}^{a}$ is:

$$
\Lambda_{22}^{a}=\left[\begin{array}{ccccc}
L_{u 1,1}+L_{t 1,1} & -L_{t 1,1} & . & 0 & 0 \\
-L_{t 1,1} & L_{l 1,1}+L_{t 1,1} & . . & 0 & 0 \\
\vdots & \vdots & \ddots & \vdots & \vdots \\
0 & 0 & . . & L_{u 2,3}+L_{t 2,3} & -L_{t 2,3} \\
0 & 0 & . . & -L_{t 2,3} & L_{l 2,3}+L_{t 2,3}
\end{array}\right] \in \mathbb{R}^{12 \times 12}
$$

Matrix $\left(\Lambda_{22}^{b}\right)^{T} \in \mathbb{R}^{4 \times 12}$ is shown in equation (7).

$$
\left(\Lambda_{22}^{b}\right)^{T}=\left[\begin{array}{cccccccccccc}
-L_{t 1,1} & L_{t 1,1} & 0 & 0 & 0 & 0 & 0 & 0 & L_{t 1,3} & L_{t 1,3} & 0 & 0 \\
0 & 0 & -L_{t 2,1} & L_{t 2,1} & 0 & 0 & 0 & 0 & 0 & 0 & L_{t 2,3} & L_{t 2,3} \\
0 & 0 & 0 & 0 & -L_{t 1,2} & L_{t 1,2} & 0 & 0 & L_{t 1,3} & L_{t 1,3} & 0 & 0 \\
0 & 0 & 0 & 0 & 0 & 0 & -L_{t 2,2} & L_{t 2,2} & 0 & 0 & L_{t 2,3} & L_{t 2,3}
\end{array}\right]
$$

On the other hand, the matrix $\Gamma(u) \in \mathbb{R}^{28 \times 28}$ has the following form:

$$
\Gamma(u)=\left[\begin{array}{cc}
\mathbf{0}_{12 \times 12} & \Gamma_{12}(u) \\
\Gamma_{21} & \Gamma_{22}
\end{array}\right] ; \Gamma_{21}=\left[\begin{array}{c}
-\mathbf{I}_{12 \times 12} \\
\mathbf{0}_{4 \times 12}
\end{array}\right] \in \mathbb{R}^{16 \times 12} ; \Gamma_{12}(u)=-U(u) \cdot C^{-1} \cdot \Gamma_{21}^{T} \in \mathbb{R}^{12 \times 16}
$$

where $U(u) \in \mathbb{R}^{12 \times 12}$ is a diagonal matrix which includes the control variables, and $C \in \mathbb{R}^{12 \times 12}$ is a diagonal matrix which includes the capacitance of each sub-module, see (9).

$$
U(u)=\operatorname{diag}\left[N_{u 1,1} \cdot n_{u 1,1}, N_{l 1,1} \cdot n_{l 1,1}, . ., N_{l 2,3} \cdot n_{l 2,3}\right] ; C=\operatorname{diag}\left[\mathcal{C}_{u 1,1}, \mathcal{C}_{l 1,1}, . ., \mathcal{C}_{l 2,3}\right]
$$


And finally, matrix $\Gamma_{22} \in \mathbb{R}^{k(3 n-1) \times k(3 n-1)}$ is equal to $-\Lambda_{22}$ by changing only the coefficients of the inductances by the respective resistances. As matrix $\Lambda$ is invertible, then is possible to write the system (4) in the following classic form:

$$
\dot{x}=A(u) x+\mathcal{B} ; \quad A(u)=\Lambda^{-1} \cdot \Gamma(u)=\left[\begin{array}{cc}
\mathbf{0} & \Gamma_{12}(u) \\
\Lambda_{22}^{-1} \cdot \Gamma_{21} & \Lambda_{22}^{-1} \cdot \Gamma_{22}
\end{array}\right]=\left[\begin{array}{cc}
0 & A_{12}(u) \\
A_{21} & A_{22}
\end{array}\right] ; \mathcal{B}=\Lambda^{-1} \cdot B \in \mathbb{R}^{28)}
$$

At this point, it is important to realize that the system is not linear, since the input $u$ is multiplied by the state $x$, but if we apply the theory of switching systems [14], we can transform the same problem in another which will be composed of a finite number of linear systems, as many as possible combinations between the control variables could appear. From equation (10), we can note as all of these possible linear system, which appear variating the input $u$, have always the same equilibrium point. This fact should not surprise us, moreover from the point of view of physics is consistent. Basically, the change in the control variables is the same as if we change the total capacity of each module. Due the structure of the system, where inductances are placed in series with the capacitors in the upper and lower arms, in steady state the current through these arms will be zero whatever the combination of control variables $u$, and therefore the voltage drop in the capacitor will always be the same. From the point of view of switched control theory the fact that all systems have the same equilibrium point, is very important in order to prove the global stability of the system and to find a global Lyapunov function, as will be explained in section 2.3.

Another important remark is that, in this study we have not used the commonly called circulating current, which is habitually used in the literature for MMC applications [12, 13]. Operation with this virtual variable, which is the supposed current that is passing through the mesh which includes upper and lower arm of the same leg. This fact is only true if the resistances and inductances are exactly the same, and it is very difficult to be true in real applications.

\subsection{Stability.}

Firstly, it is straightforward to verify that matrix $\Lambda$ shown in (5) is invertible. Moreover we are going to prove that it is positive definite. We proceed as follows, equation (5) could be rewritten as:

$$
\Lambda=\left[\begin{array}{cc}
I_{2 n k \times 2 n k} & \mathbf{0} \\
\mathbf{0} & \Lambda_{2,2}
\end{array}\right]=\left[\begin{array}{ccc}
I_{2 n k \times 2 n k} & \mathbf{0} & \mathbf{0} \\
\mathbf{0} & \Lambda_{2,2}^{a} & \Lambda_{2,2}^{b} \\
\mathbf{0} & \left(\Lambda_{2,2}^{b}\right)^{T} & \Lambda_{2,2}^{c}
\end{array}\right]
$$

therefore, it is clear that if, and only if $\Lambda_{2,2}$, defined in equation (6), is positive definite then matrix $\Lambda$ is positive definite also. The next Lemma 1 and Lemma 2 will give us sufficient conditions for achieve that matrix $\Lambda_{22}$ will be positive definite.

Lemma 1 Let $n, l \in \mathbb{N}^{*}, A_{11} \in \mathbb{R}^{n \times n}, A_{12} \in \mathbb{R}^{n \times l}, A_{21} \in \mathbb{R}^{l \times n}, A_{22} \in \mathbb{R}^{l \times l}$ and

$$
A=\left[\begin{array}{ll}
A_{11} & A_{12} \\
A_{21} & A_{22}
\end{array}\right] \in \mathbb{R}^{(n+l) \times(n+l)},
$$

$A$ is positive definite if, and only if, $A_{22}-A_{21} \cdot A_{11}^{-1} \cdot A_{12}$ is positive definite and $A_{11}$ is positive definite (or equivalently, if, and only if $A_{22}$ is positive definite and $A_{11}-A_{12} \cdot A_{22}^{-1} \cdot A_{21}$ is positive definite).

Lemma 2 Let $n, k \in \mathbb{N}^{*}$, let $A \in \mathbb{R}^{(n k) \times(n k)}$ be a matrix defined as follows:

$$
A=\left[\begin{array}{cccc}
A_{1} & B & . . & B \\
B & A_{2} & . . & B \\
\vdots & \vdots & \ddots & \vdots \\
B & B & . . & A_{n}
\end{array}\right]
$$

where $A_{i} \in \mathbb{R}^{k \times k}$ is defined as $A_{i}=\operatorname{diag}\left(\alpha_{i}^{1}, . ., \alpha_{i}^{k}\right) \forall i \in\{1, . . n\}$ and $B \in \mathbb{R}^{k \times k}$ equals to $B=\operatorname{diag}\left(\beta^{1}, . ., \beta^{k}\right)$. If $\forall i \in\{1, . . n\}$, and $\forall j \in\{1, . . k\}, \alpha_{i}^{j}>\beta^{j} \geq 0$ then $A$ is positive definite. 


\subsubsection{Equilibrium points.}

Now we study the form of the equilibrium points, and we will see for any different input $u$ all the systems have the same equilibrium point, as discussed before.

In the equilibrium $\left(x^{*}\right)$, if we define:

$$
x^{*}=\overbrace{\left[. ., v_{u i, j}^{*}, v_{l i, j}^{*}, . ., . ., i_{u i, j}^{*}, i_{l i, j}^{*}, . ., i_{p 1.1}^{*}, . ., i_{p k, 1}^{*}, . ., i_{p k, n-1}^{*}\right.}^{12}]^{T}=\overbrace{x_{v}^{*}}^{12} \overbrace{x_{i}^{*}}^{12} \overbrace{x_{i p}^{*}}^{4}]^{T}
$$

from systems (4) and (10), and for any bounded input $u \neq 0$, it is clear that:

$$
\left\{\begin{array}{c}
-\Lambda_{22}^{-1} \cdot \Gamma_{22}\left[\begin{array}{c}
x_{i}^{*} \\
x_{i p}^{*}
\end{array}\right]+\Lambda_{22}^{-1} \cdot\left[\begin{array}{l}
I \\
\mathbf{0}
\end{array}\right] \cdot x_{v}^{*}-\Lambda_{22}^{-1} \cdot\left[\begin{array}{l}
V \\
\mathbf{0}
\end{array}\right]=\mathbf{0} \\
U(u) \cdot C^{-1} \cdot x_{i}^{*}=\mathbf{0}
\end{array}\right.
$$

where $V=\left[. ., V_{D C i}, V_{D C i}, . .\right]^{T} \in \mathbb{R}^{2 n k}$ in concordance with equation (4). From (14) as $\left.\exists(U(u))\right)^{-1}$ if $u \neq 0$, then $x_{i}^{*}=0$, and consequently:

$$
-\Gamma_{22}\left[\begin{array}{c}
\mathbf{0} \\
x_{i p}^{*}
\end{array}\right]+\left[\begin{array}{c}
x_{v}^{*} \\
\mathbf{0}
\end{array}\right]=\left[\begin{array}{c}
V \\
\mathbf{0}
\end{array}\right] ; \text { as } \Gamma_{22}=\left[\begin{array}{cc}
\Gamma_{22}^{a} & \Gamma_{22}^{b} \\
\left(\Gamma_{22}^{b}\right)^{T} & \Gamma_{22}^{c}
\end{array}\right] \Rightarrow \Gamma_{22}^{c} \cdot x_{i p}^{*}=\mathbf{0} \Rightarrow x_{i p}^{*}=\mathbf{0} \Rightarrow x_{v}^{*}=V
$$

Summarizing, for the system (4) and for any input $u$ all the equilibrium points have always the form: $x_{v}^{*}=V, x_{i}^{*}=\mathbf{0}$ and $x_{i p}^{*}=\mathbf{0}$

\subsubsection{Stability.}

The follow theorem 1 summarizes the stability proposed for of this MMC converter for a general case with $k$ phases and $n$ terminals.

Theorem 1 The converter described by (4) is globally exponentially stable for any bounded control variable defined in (4).

Proof.- With the same reasoning as for $\Lambda$, we can prove that $\Gamma(u)$ is always negative definite for any $u$ bounded, since the elements of $\Gamma_{22}$ have opposite sign than the $\Lambda_{22}$ ones.

All possible systems that can exist, which are derived from all possible combinations for the control variables $u$, have the same point of equilibrium as has been shown above. Consequently, if we find a global Lyapunov function for all of them, the theorem will be prove.

The matrix $A(u)$ of the system (4) could be rewritten as:

$$
A(u)=\Lambda^{-1} \cdot \Gamma(u)=\left[\begin{array}{cc}
\mathbf{0} & -C^{-1} \cdot U(u) \cdot \Gamma_{21}^{T} \\
\Lambda_{22}^{-1} \cdot \Gamma_{21} & \Lambda_{22}^{-1} \cdot \Gamma_{22}
\end{array}\right] ; \Lambda_{22}^{-1}=\left[\begin{array}{cc}
\mathcal{M}^{a} & \mathcal{M}^{b} \\
\left(\mathcal{M}^{b}\right)^{T} & \mathcal{M}^{c}
\end{array}\right]
$$

where $\mathcal{M}^{a}$ and $\mathcal{M}^{c}$ are symmetric and positive definite matrices.

If we suppose that the equilibrium point of system (4) could be rewritten as: $x^{*}=-A(u)^{-1} \cdot \mathcal{B}$, and if we define, $\tilde{x}=x-x^{*}$, then the system (4) becomes to: $\dot{\tilde{x}}=A(u) \cdot \tilde{x}$.

If we consider the Lyapunov function shown in (17), is clearly a positive definite function since $P$ is positive definite by means of Lemma 1 and Lemma 2.

$$
\tilde{\mathcal{V}}=\tilde{x}^{T} P \tilde{x} ; \quad P=\left[\begin{array}{ccc}
U^{-1} \cdot C & \varepsilon I & \mathbf{0} \\
\varepsilon I & \Lambda_{22}^{a} & \Lambda_{22}^{b} \\
\mathbf{0} & \left(\Lambda_{22}^{b}\right)^{T} & \Lambda_{22}^{c}
\end{array}\right]
$$

Therefore, $\tilde{\mathcal{V}}$ is always positive $\forall \tilde{x} \neq 0$. The parameter $\varepsilon$ is chosen as $\varepsilon^{2}<\lambda_{0} \mathcal{C}_{0} / u_{m}$, where we have taken into account that $\lambda_{0}=\min \left(\sigma\left(\Lambda_{22}\right)\right){ }^{1}, \mathcal{C}_{0}$ is the minimum value of sub-module capacitors, and $u_{m}$ is the maximum value of the control variable $u$.

\footnotetext{
${ }^{1} \sigma(A)$ is the spectrum of $\mathrm{A}$.
} 
On the other hand:

$$
P A+A^{T} P=\left[\begin{array}{cc}
-2 \cdot \varepsilon \cdot \mathcal{M}^{a} & \varepsilon \cdot\left[\mathcal{M}^{a} \mathcal{M}^{b}\right] \cdot \Gamma_{22} \\
\varepsilon \cdot \Gamma_{22} \cdot\left[\begin{array}{c}
\mathcal{M}^{a} \\
\left(\mathcal{M}^{b}\right)^{T}
\end{array}\right] & 2 \cdot\left(\Gamma_{22}+\varepsilon \cdot\left[\begin{array}{cc}
U \cdot C^{-1} & \mathbf{0} \\
\mathbf{0} & \mathbf{0}
\end{array}\right]\right)
\end{array}\right]
$$

and applying Lemma 1 it holds that $\exists \delta>0$ such that if $0<\varepsilon<\delta$ the matrix $A P+P^{T} A$ shown in (18) is negative definite, and consequently the time derivate of Lyapunov function defined in (17) is always negative. Consequently, for any control variable $u$, the Lypaunov function defined in (17) is positive definite and its time derivate is always negative definite. This means that the the system defined in (4) is asymptotically stable.

\subsection{Sinusoidal signal generation}

If we connect gradually the capacitors in sequence, a sinusoidal voltage will appear between two phases as shows figure 2. It is considered that the signals for the first arm are used as reference for the other arms of the same terminal. In figure 2 appears the parameter $m$, which is defined as the phase difference between the pulse control signals of the capacitors in one arm (it is noteworthy that duty cycle in each signal is always $50 \%$ ). This value depends on the number of sub-modules per arm. On the other way, the parameter $\mu$ is the phase difference between the control signal for different arms and it depends on the number of arms in each terminal.

In this way, and from the viewpoint of control, only one insertion index value is required by terminal in order to govern the converter. In equations (19) both parameters are defined, where $T$ is the period of the sinusoidal signals, $N$ is the number of sub-modules per arm, and $k$ is is the number of phases per terminal.

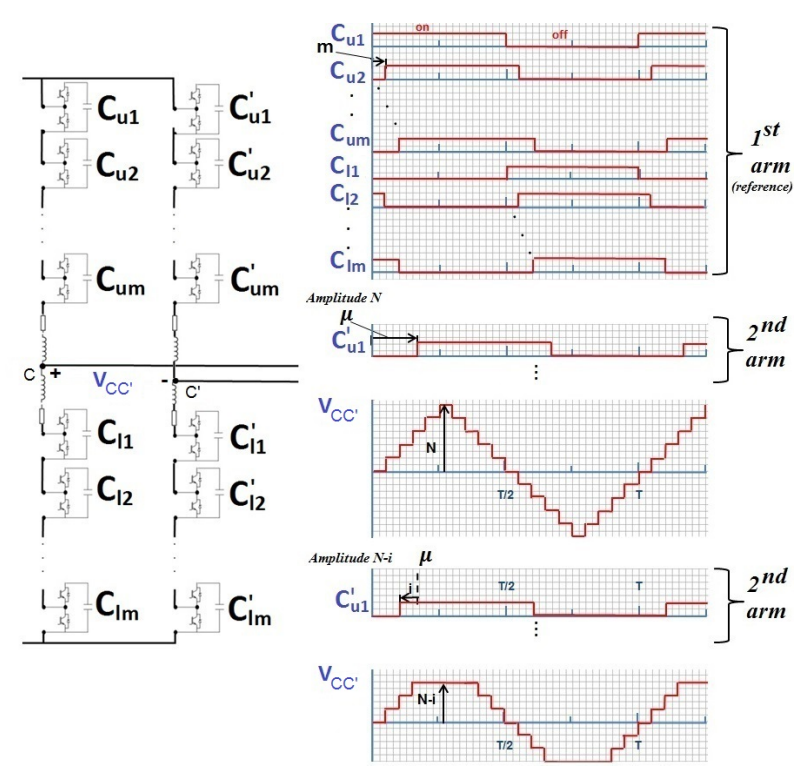

Figure 2: Sinusoidal signal generation.

$$
m \in \mathbb{R}, \quad 0 \leq m \leq \frac{T}{2} \quad \text { s.t } m=\frac{T / 4}{N} ; \mu \in \mathbb{R}, 0 \leq \mu \leq \frac{T}{2} \quad \text { s.t } \quad \mu=\frac{T}{k}
$$

If we want to obtain a phase voltage $\left(V_{a b}\right)$ with an amplitude equal to $N-i$, where $i \in \mathbb{N}$, then we have to advance the pulse signal for the corresponding arm $i$ intervals of length $m$ with respect to value $\mu$, which corresponds for a phase voltage $\left(V_{a b}\right)$ with an amplitude equals to $N$, as figure 2 shows. In this way, we have achieved that all capacitors are always used the same amount of time. Also, we can remark that in the phase voltage appears a landing whose length is equal to $(i+1) m$, but the value for the first 
harmonic of this signal is always displaced the value $\mu$ with respect to the reference. In consequence, the obtained phase voltages at each terminal are uniformly separated according to the number of arms that there exists, as shown equation (20):

$$
V_{a b}=n_{j} \cdot V_{D C, j} \cdot \sin \left(\omega \cdot t+\frac{2 \pi}{k}\right)
$$

where $n_{j}$ is the insertion index and $V_{D C, j}$ is the DC voltage of the grid $j$.

The reached voltage has not a pure sinusoidal form, but if we consider sufficient sub-modules, it can be approximated by a sine. When the number of sub-modules is not big enough, it is interesting to study the harmonics of the created signals, in order to improve the behaviour of the converter. In subsection 2.6 a discussion in order to improve the harmonics is presented.

\subsection{Balancing}

Although all capacitors are connected the same amount of time, it is also important the order of the connection in each cycle, since the instantaneous currents are not the same for each interval of each cycle. Therefore, depending on the state of charge of each capacitor $\left(W_{C}\right)$, the sequence will vary in each cycle. The less charged capacitor will be connected in the first interval, and the more charged in the last interval of each cycle. Thanks to this procedure, we can achieve that all capacitors are kept always charged with close values between them.

\subsection{Harmonics study}

The problem of the harmonics for AC systems causes many disadvantages, including lower quality of the signal and therefore greater losses. In addition, for electronic devices, due to their discontinue nature the problem is increased. Therefore it is interesting to study them, especially if the number of modules is not very high.

A comparison between two control philosophies for the switches is shown. The first one considers that the turn on of them have a constant separation (variable $m$ defined above). In the second the turn on is governed by a law in order to achieve that the first harmonic of the generated signal will have unity amplitude and the amplitude of the high order harmonics will be small. The idea is as follows, if we consider that we have $N$ sub-modules, then we have $N$ steps. If we define $a_{1}$ as the time necessary to achieve the value $1 / N$ of the amplitude of the sine as shows figure 3 , we can chose $d_{1}$ in the form that the area enclose by the sine function between 0 and $a_{1}$ (the green area in figure 3 ), will be the same as the area of rectangle with sides $a_{1}-d_{1}$ and $1 / N$ (the hatched area in figure 3 ). Proceeding in analogous form, we can obtain the general terms as show equations (21).

$$
\begin{gathered}
\sin \left(\frac{2 \pi}{T} a_{1}\right)=\frac{1}{N} \Rightarrow a_{1}=\frac{T}{2 \pi} \arcsin \left(\frac{1}{N}\right) \Rightarrow \int_{0}^{a_{1}} \sin \left(\frac{2 \pi}{T} \cdot t\right) d t=\frac{a_{1}-d_{1}}{N} \Rightarrow d_{1}=\frac{T}{2 \pi} \cdot\left[\arcsin \left(\frac{1}{N}\right)-\left(N-\sqrt{N^{2}-1}\right)\right] \\
\sin \left(\frac{2 \pi}{T} a_{2}\right)=\frac{2}{N} \Rightarrow \ldots \Rightarrow \int_{a_{1}}^{a_{2}} \sin \left(\frac{2 \pi}{T} t\right) d t=\frac{d_{2}-a_{1}}{N}+2 \frac{a_{2}-d_{2}}{N} \Rightarrow d_{2}=\frac{T}{2 \pi}\left[2 \cdot \arcsin \left(\frac{2}{N}\right)-\arcsin \left(\frac{1}{N}\right)-\left(\sqrt{N^{2}-1}-\sqrt{N^{2}-4}\right)\right] \\
\vdots \\
\sin \left(\frac{2 \pi}{T} a_{N}\right)=\frac{N}{N} \Rightarrow \ldots \Rightarrow d_{p}=\frac{T}{2 \pi}\left[p \cdot \arcsin \left(\frac{p}{N}\right)-(p-1) \arcsin \left(\frac{p-1}{N}\right)-\left(\sqrt{N^{2}-(p-1)^{2}}-\sqrt{N^{2}-p^{2}}\right)\right]
\end{gathered}
$$

In table I we can observe the different harmonics of both philosophies for $N=5$. Another important

Table I: Harmonic amplitudes

\begin{tabular}{|l|c|c|c|c|c|c|c|c|}
\hline & \multicolumn{10}{|c|}{ Harmonics order } \\
\hline & 1 & 3 & 5 & 7 & 9 & 11 & 13 & 15 \\
\hline Mode 1 & 0.82 & 0.093 & 0.036 & 0.020 & 0.014 & 0.012 & 0.011 & 0.012 \\
\hline Mode 2 & 1 & 0.008 & 0.009 & 0.004 & 0.005 & 0.016 & 0.020 & 0.007 \\
\hline
\end{tabular}

consideration is that with both philosophies the duty cycle for each switch is always $50 \%$. 


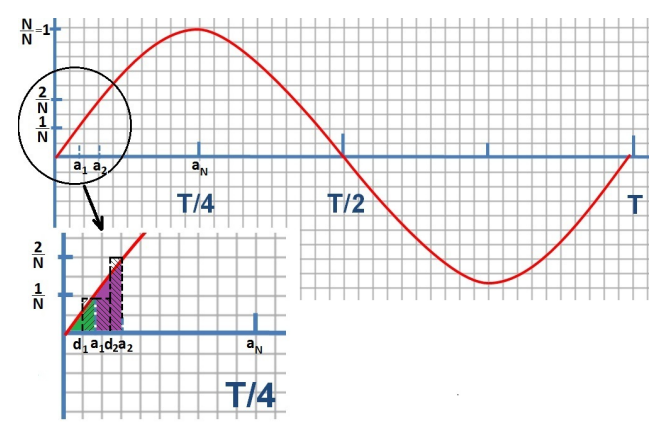

Figure 3: Harmonic construction.

\subsection{Power transport explanation}

The power transfer between terminals could be explained as follows. Due to all voltages at each terminal between equivalent phases are in phase and have the same frequency, then the power direction in each terminal will depend on the amplitude of voltages, or equivalently, the insertion indices. Therefore the power goes to the higher voltage values to lower voltages.

However, another important explanation is required. In order to the energy can flow in all directions for all terminals, it is very important the relation between the different values of the DC voltage in each external network as well as the number of sub-modules. If we assume that the system is well balanced in steady-state, the voltage in each capacitor of each sub-module will be as shown the equation (22):

$$
u_{C, u l, i}=\frac{V_{D C, i}}{N}
$$

so depending on the insertion index and the values of the voltage in each capacitor, it could be possible to transmit power in any direction, it is even possible to transmit power from networks with smaller DC voltage to other networks with higher DC values. In fact, the relationship that must be satisfied to transmit power from terminal $i^{\text {th }}$ to $j^{t h}$ is shown in equation (23). The key issue is the amplitude of the AC internal generated signals.

$$
u_{C, u l, i} \cdot n_{i}=\frac{V_{D C, i} \cdot n_{i}}{N}>\frac{V_{D C, j} \cdot n_{j}}{N}
$$

Also it could be noted that the best performance is obtained when the relationship between the different DC voltages of each network are closer between them, because the losses are smaller.

\section{Simulations}

A three terminal converter with two phases based in MMC technology, shown in figure 1 is tested in order to verify the proper operation of the control philosophy, which has been explained in section 2.3

The voltage of each DC grid are: $V_{D C, 1}=5 \mathrm{kV}, V_{D C, 2}=6.25 \mathrm{kV}$ and $V_{D C, 3}=7.5 \mathrm{kV}$. The main values of MMC are shown in table II.

Table II: Simulation parameter values

\begin{tabular}{|c|c|c|}
\hline \multicolumn{3}{|c|}{ MMC parameters } \\
\hline$R_{1}$ & Resistance arm 1 & $0.1 \Omega$ \\
\hline$R_{2}$ & Resistance arm 2 & $0.1 \Omega$ \\
\hline$L_{1}$ & Inductance arm 1 & $0.01 \mathrm{H}$ \\
\hline$L_{2}$ & Inductance arm 2 & $0.01 \mathrm{H}$ \\
\hline$C$ & Total module capacitance & $0.01 \mathrm{~F}$ \\
\hline$f$ & AC signals frequency & $100 \mathrm{~Hz}$ \\
\hline
\end{tabular}




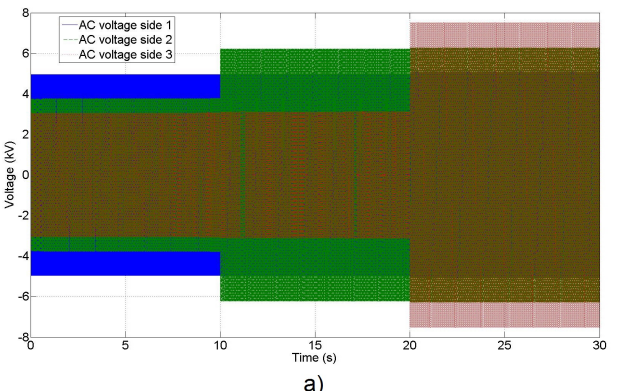

a)

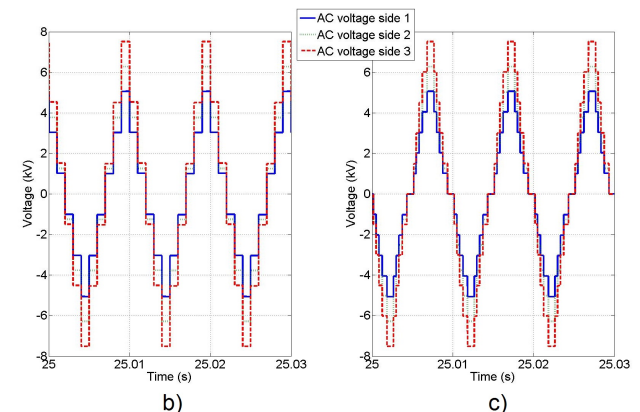

Figure 4: a) AC generated signal voltage b) Voltages for strategy 1 b) Voltages for strategy 2.

We can observe in figure $4 \mathrm{a}$ as during interval $0 \leq t \leq 10$, the peak value of the voltage in terminal 1 is $5 \mathrm{kV}\left(n_{1}=1\right), 3.75 \mathrm{kV}\left(n_{2}=0.6\right)$ in terminal 2 and $3 \mathrm{kV}\left(n_{3}=0.4\right)$ in terminal 3 . Consequently, terminal 1 provides energy to terminals 2 and 3. In interval $10 \leq t \leq 20$, the first and the second one supply the energy to terminal 3 because the voltages are $5 \mathrm{kV}\left(n_{1}=1\right), 6.25 \mathrm{kV}\left(n_{2}=1\right)$ and $3 \mathrm{kV}$ $\left(n_{3}=0.4\right)$ respectively for each terminal. Finally during $20 \leq t \leq 30$, as the voltage between points $\mathrm{M}$ and $\mathrm{M}^{\prime}$ is equals to voltage in node 2 , the terminal 3 provides the energy only to the terminal 1 , and the terminal 2 does not absorb or supply energy. The voltages in this last interval are $5 \mathrm{kV}\left(n_{1}=1\right), 6.25 \mathrm{kV}$ $\left(n_{2}=1\right)$ and $7.5 \mathrm{kV}\left(n_{3}=1\right)$ respectively for each terminal.

In figures $4 \mathrm{a}$ and $4 \mathrm{~b}$ we observe with more precision the generated voltages with both strategies explained in 2.6. We observe as with strategy 2 the signals have a form more similar to a sine than with strategy 1 . In figures $5 \mathrm{a}$ and $5 \mathrm{~b}$ the phase currents for terminal 1 are shown.

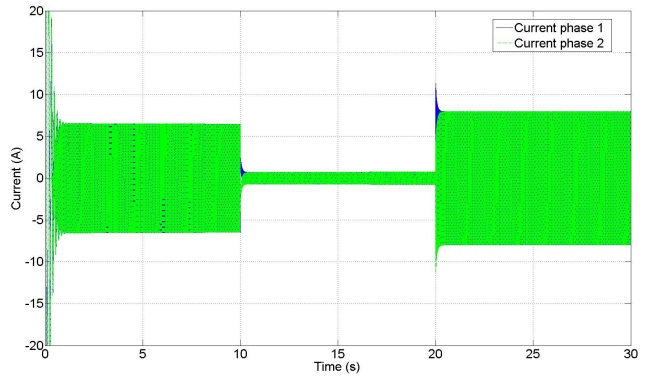

a)

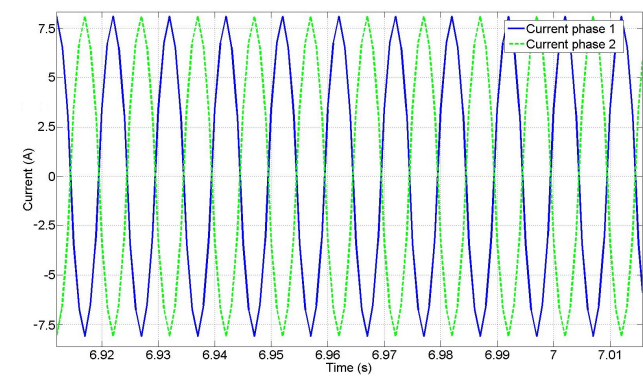

b)

Figure 5: a) Phase currents terminal 1 b) Phase currents terminal 1. Zoom.

Finally in figure 6a, we observe as all capacitors for each terminal remains always with the same voltage according to the strategy adopted. In accordance with equation (22) we can remark that the voltages for each capacitor are $1 \mathrm{kV}, 1.25$ and $1.5 \mathrm{kV}$ respectively for each terminal. Figure $6 \mathrm{~b}$ shows the state of charge of the capacitors from the upper arm in the first terminal and first phase, we can appreciate as the balancing philosophy is applied.
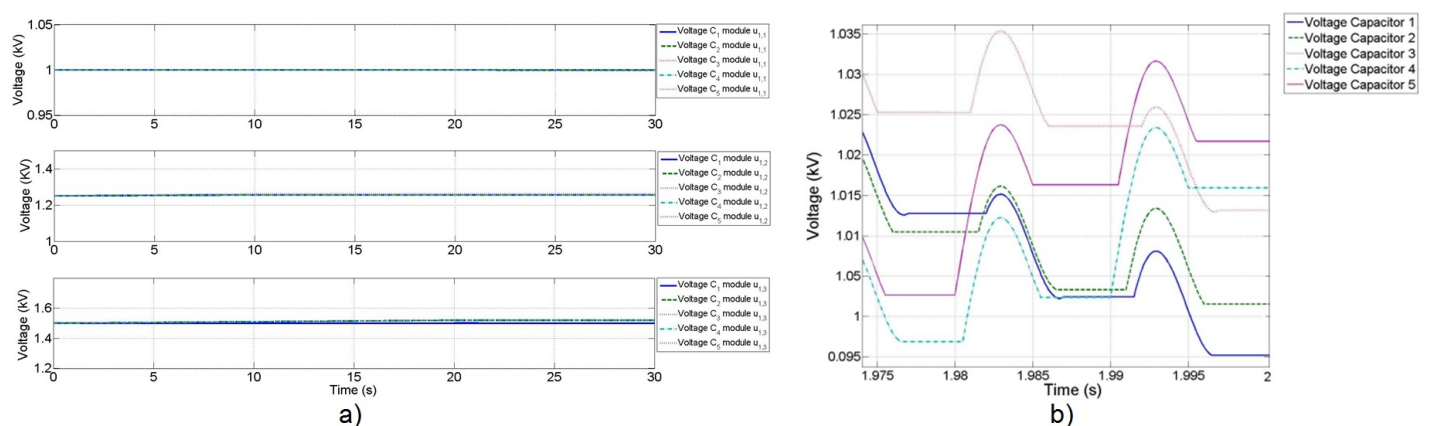

Figure 6: a) Capacitors state of charge b) Capacitors of the upper arm in the first terminal and first phase. 


\section{Conclusions}

In this paper a multi-terminal DC/DC converter with 2 phases and 3 terminals is discussed using MMC topology. A mathematical proof is given in order to guarantee the stability of the system. Although the proof is valid for any values of DC voltages, in a real implementation the voltage relations between DC sides will most likely be small in order to kept small losses. Anyway, and thanks to the simulations we can conclude that the proposed control is valid for the use of these types of converters.

In addition, a deep study on how to improve the harmonics in the generated signals is shown. Also a philosophy of balancing is implemented in order to favour the use of capacitors in each module in a well-adjusted way.

\section{References}

[1] J. Arrillaga, Y. Liu, and N. Watson, Flexible Power Transmission. The HVDC Options. John Wiley \& Sons Ltd, 2007.

[2] S. Johansson, G. Asplund, E.Jansson, and R. Rudervall, "Power system stability. benefits with VSC-HVDC transmission systems," CIGRE, 2007.

[3] A. Lesnicar and R. Marquardt, "An innovative modular multilevel converter topology suitable for a wide power range," in Power Tech Conference Proceedings, 2003 IEEE Bologna, vol. 3, June 2003.

[4] U. Gnanarathna, A. Gole, and R. Jayasinghe, "Efficient modeling of modular multilevel hvdc converters (MMC) on electromagnetic transient simulation programs," Power Delivery, IEEE Transactions on, vol. 26, no. 1, pp. 316-324, Jan 2011.

[5] G. Bergna, A. Garces, E. Berne, P. Egrot, A. Arzande, J.-C. Vannier, and M. Molinas, "A generalized power control approach in abc frame for modular multilevel converter HVDC links based on mathematical optimization," Power Delivery, IEEE Transactions on, vol. 29, Feb 2014.

[6] M. Jiménez Carrizosa, J. Cortés, A. Benchaib, P. Alou, G. Damm, J. A. Cobos, and F. LamnabhiLagarrigue, "DC/DC converters as DC circuit-breakers in HVDC networks operation." in Power Electronics and Applications (EPE), 2014 16th European Conference on, August 2014, pp. 1-10.

[7] G.Ortiz, J. Biela, D. Bortis, and J. Kolar, "1 MW, $20 \mathrm{kHz}$, isolated, 1.2-12kV bidirectional DC-DC renewable converter for energy applications," Int. Power Electronics Conference, IEEE, 2010.

[8] M. Jiménez Carrizosa, A. Benchaib, P. Alou, and G. Damm, "DC transformer for DC/DC connection in HVDC network," in 15th European Conference on EPE, Sept 2013.

[9] G.Ortiz, J. Biela, and J. Kolar, "Optimized design of medium frequency transformers with high isolation requirements," 36th Conference on IEEE Industrial Electronics Society, November 2010.

[10] T. Lüth, M. Merlin, T. Green, C. Barker, F. Hassan, R. Critchley, R. Crookes, and K. Dyke, "Performance of a dc/ac/dc VSC system to interconnect HVDC systems," in AC and DC Power Transmission (ACDC 2012), 10th IET International Conference on, Dec 2012, pp. 1-6.

[11] J. Stevens and D. Rogers, "Control of multiple VSC-HVDC converters within an offshore AC-hub," in Energytech, 2013 IEEE, May 2013, pp. 1-5.

[12] G. Kish, M. Ranjram, and P. Lehn, "A modular multilevel DC/DC converter with fault blocking capability for HVDC interconnects," Power Electronics, IEEE Transactions on, vol. 30, Jan 2015.

[13] L. Harnefors, A. Antonopoulos, S. Norrga, L. Angquist, and H.-P. Nee, "Dynamic analysis of modular multilevel converters," Industrial Electronics, IEEE Transactions on, vol. 60, July 2013.

[14] D. Liberzon, Switching in systems and control. Birkhauser, 2003. 